\title{
The plastic instability behaviour of laser-textured steel sheet
}

\author{
Huan Shen*, Guang-Nan Chen, Guo-Chen Li \\ Institute of Mechanics, Academia Sinica, Beijing 100080, People's Republic of China \\ Received 9 February 1996; revised 14 May 1996
}

\begin{abstract}
As the production of a new technique that can offer both good formability and high image clarity for texturing metal sheet, laser-textured sheet has attracted the attention of many manufacturers and users. Among the many subjects to be studied, plastic instability behaviour of the laser-textured sheet is one of most important to understand its ability in extending material ductility and to appropriately control this technique. Experimental investigations are carried out in this paper to study the macroscopic behaviour and microstructural mechanism of the laser-textured sheet, and comparison is made with the normal sheet taken from the same coil of metal sheet. It is demonstrated that, the difference in the behaviour of plastic instability obviously shows tendency to delay strain localization and the onset of thickness necking. Shear banding and internal void damage are spread to a much wider region in the sheet being laser-textured. The prestrained microcraters enforced on the surface of the textured sheet act as hardening spots, which are likely to share out deformation and inhibit the increasing rate of voiding, and eventually favouring the ductility of the material used.
\end{abstract}

Keywords: Laser-texturing; Instability; Voids; Steel sheet

\section{Introduction}

It is well known that the stretch forming of metal sheets involves a large amount of straining such that plastic instability in tension leads to eventual failure. The instability of metal sheet and its fracture behaviour are affected by many factors, such as the initial inhomogeneity of sheet metal, the process of damage evolution, the change in stress ratio, normal anisotropy, strain-rate sensitivity, the gradient of strain subjected to a locally deforming region, and the triaxial state of stress in the necking zone [1-4]. Taking appropriate control and adjustment of these factors can provide the possibility of improving or enhancing the stability of deformation.

In recent years, a new technique for texturing the roll surface by using laser pulses has been developed and applied successfully to steel sheet production. Compared with other techniques, such as shot-blast texturing as well as electric discharge texturing, laser texturing has the advantages of offering an appropriate surface pattern and the easy handling in technique. The

\footnotetext{
* Corresponding author.
}

laser texturing initially forms a regular and periodic pattern on the surface of the roll to be used, then afterwards this regular pattern is inversely enforced on the surface of steel sheets during the process of cold rolling and temper rolling. Owing to the fact that the laser-textured sheet (LTS) has good formability and high image clarity, this new technique has attracted the attention of many steel sheets manufacturers and users. Several investigations have been published with regard to this topic [5-7]. They concentrated their attention to the effect of surface roughness on the friction behaviour of the LTS, or to its image clarity for paint-coated surface and press formability [5,6]. However, to the knowledge of the authors, the plastic instability behaviour of the LTS and its internal mechanism that yields the improvement of ductility are so far not being reported.

In the present paper, the experimental investigation carried out on LTS of a low carbon steel is carefully arranged to estimate the potential of using this tech-. nique to delay the onset of localized necking. Also, it is to demonstrate the characteristics of the instability behaviour of the LTS with its peculiar manner of void development. The results would serve as the basis for better understanding the macroscopic behaviour of 
laser-textured sheet metal and also for correlating this behaviour to the microstructural mechanism of the material.

\section{Experimental procedure}

\subsection{Material preparation}

The material used in this study is a rimmed low-carbon steel sheet. The chemical composition of this steel (wt. \%) is $0.08 \mathrm{C}, 0.30 \mathrm{Mn}, 0.04 \mathrm{~S}, 0.04 \mathrm{P}$, balance Fe. A coil of annealed $0.4 \mathrm{~mm}$ thick sheet was divided into two sets for different processes. One was cold rolled $12.5 \%$ to a thickness of $0.35 \mathrm{~mm}$ by the laser-textured rolls, while another set was cold rolled to the same thickness but using the normal sanded rolls. The microstructures of both test sheets are composed of ferrite and free carbide.The average grain size of the ferrite is measured to have $0.028 \mathrm{~mm} \times 0.012 \mathrm{~mm}$ and the inclusion content is $0.02 \%$. Scanning electron microscope (SEM) micrographs are presented in Fig. 1(a) and (b), to demonstrate the surface features of the two sets of tested sheets. Fig. 1(a) shows the continuous rolling marks on the surface of the normal sheet (NS), while in Fig. 1(b), it can be seen that, there are many microcraters arranged regularly and indented on the surface of the steel sheet being laser-textured during cold rolling.

The diameter of microcrater is approximately 200 $\mu \mathrm{m}$, and the spacing between the centres of microcrater is approximately $250 \mu \mathrm{m}$. The morphological feature of these microcraters can be characterized by the geometrical specifications of a ring-like valley. The depth of this valley runs to $5 \mu \mathrm{m}$, and the width of the ring spreads approximately $40 \mu \mathrm{m}$. The average roughness, $R_{\mathrm{a}}$, is quantified as $1.2 \mu \mathrm{m}$ for the LTS, but is lowered to $0.7 \mu \mathrm{m}$ for the NS.

\subsection{Measurements of deformation and void growth}

The tensile specimens made for the two sets of steel sheets were machined along the rolling direction of the sheets. Each specimen was shaped as demanded by the conventional tension testing for sheet metal. The parallel length was $75 \mathrm{~mm}$, and its width was $12.5 \mathrm{~mm}$. The tensile tests were performed on an Instron 1195 universal testing machine at a cross-head speed of $1 \mathrm{~mm}$ min $^{-1}$. Loads and extensions were recorded on a chart moving at a constant speed.

In order to investigate the evolution of voiding during the whole deformation history, separate specimens of the same size as stated above were used to be deformed to different strain levels up to the stage of having local necking. These tensile specimens were unloaded after being deformed to the specified strain, then
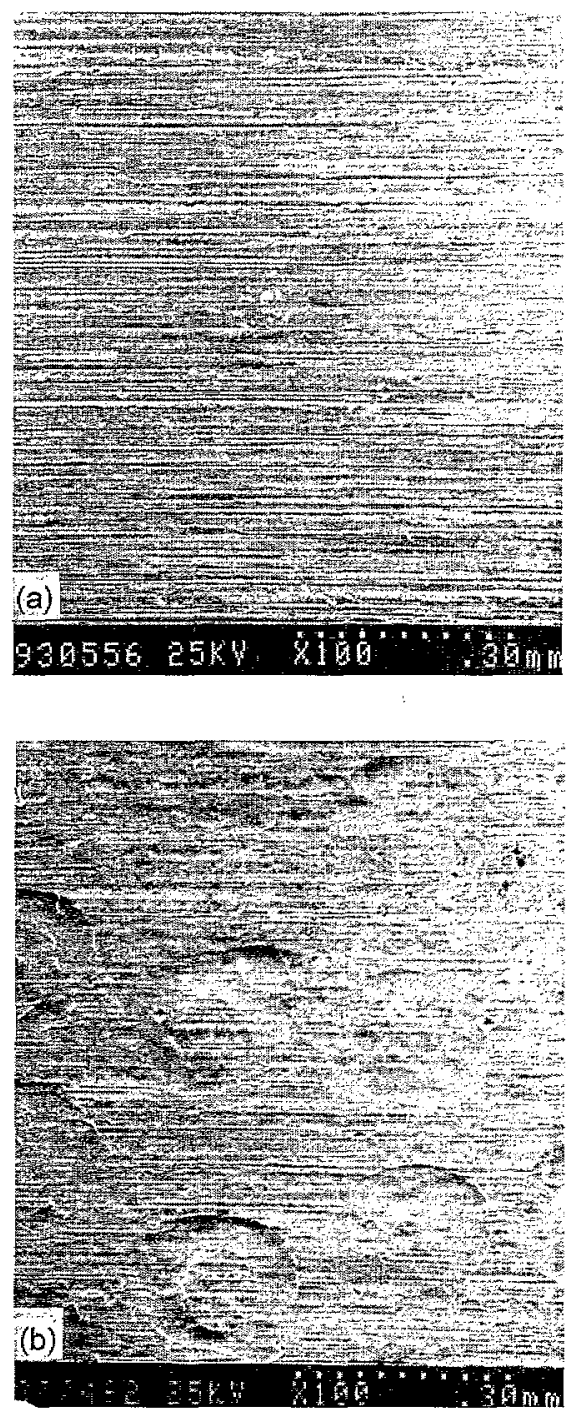

Fig. 1. The initial surface features of (a) NS and (b) LTS.

the specimens were sectioned longitudinally and polished. Metallographic analysis of the void structure was made on the longitudinal section within the gauge length, and a series of photographs were taken by using a Hitachi S570 SEM. Based on these photographs, quantitative measurements of void density, size and distribution were performed by a Q520 image analyzer. The schematic diagram of the sampling region and position taken for quantitative measurement of voids is shown in Fig. 2.

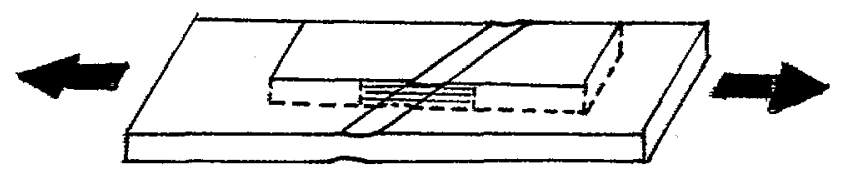

Fig. 2. Schematic demonstration of the sampling position. Shaded area corresponds to the region taken for quantitative measurement of voids. 


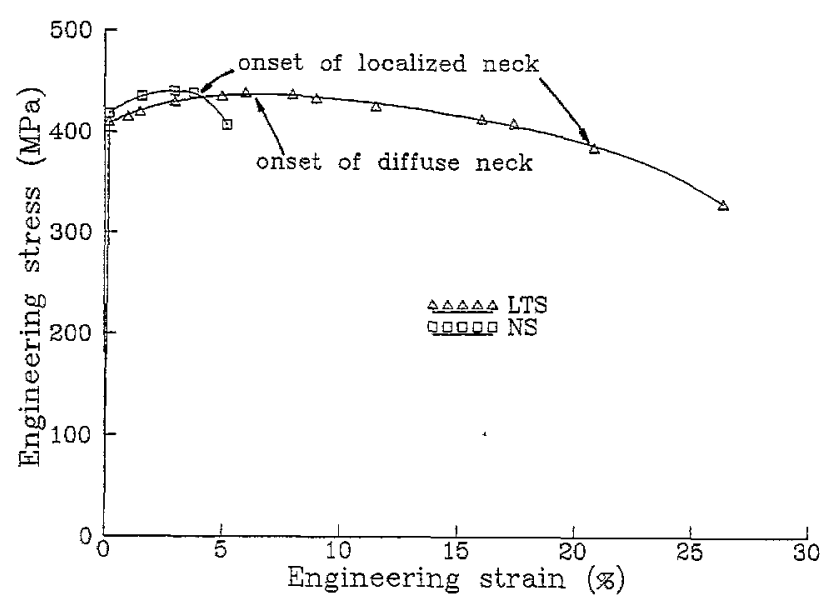

Fig. 3. Engineering stress-strain curves of both test sheets.

In situ uniaxial tensile tests were also carried out by employing the Hitachi S570 SEM as being equipped with a tensile stage, so as to observe the evolution of the morphological feature and the slip bands on the surface of the specimens during deformation. In this case, small size specimens (gauge length $=10 \mathrm{~mm}$, width $=4 \mathrm{~mm}$ ) had to be used as required by the testing equipment.

\section{Experimental results}

\subsection{The tensile behaviour of the two sets of test sheets}

Let the gauge length, width and thickness be $L_{0}, W_{0}$ and $T_{0}$, respectively. The loading force is called $F$ and the initial cross-section is $A_{0}$. The engineering stressstrain $\left(F / A_{0}-\Delta L / L_{0}\right)$ curves of the two sets of sheets are plotted in Fig. 3. During tensile testing the deformation is nearly uniform prior to the maximum load, beyond this point deformation becomes nonuniform and gradually concentrates to the middle portion of the specimen. In order to represent the tensile behaviour of the sheets in both the uniform and, most importantly, the postuniform stage of deformation, in this paper, all the elongations of tensile specimens are calculated on the basis of $10 \mathrm{~mm}$ gauge length which includes the region containing the instability bands. From Fig. 3 , it can be seen that, the engineering strain $\left(\Delta L / L_{0}\right)$ corresponding to the maximum load is approximately 0.03 in the NS and attains 0.06 in the LTS. With continued straining beyond the maximum loading point, the load drops off quickly in the NS but slowly in the LTS. This indicates that, the loss of load carrying capacity is in a smooth manner in the LTS while it is dramatic in the case of the NS specimen. In addition, the engineering strain associated with the onset of localized neck is 0.04 in the NS, but it can attain a level of 0.21 in the LTS. This fact indicates that, the limit strain of LTS is several times larger than that of the NS.

During tensile testing, the current thickness and width of the specimen were measured with a micrometer after each loading step until local necking developed. The variations of the thickness and the width, with respect to the increase of engineering strains, are shown in Fig. 4. Beyond the engineering strain $\Delta L$ / $L_{0}=0.03$, which is associated with the maximum load of the NS, the thinning of the specimen increases exponentially until localized necking takes place while the width variation is only keeping at a linear pace with respect to the straining (see Fig. 4(a)). In the LTS specimens, the engineering strain at the maximum load is $\Delta L / L_{0}=0.06$ and the variation of the width behaves in an opposite manner by showing larger shrinking than that of the thickness before the onset of localized necking (at $\Delta L / L_{0}=0.21$ ). After that, the decrease of the thickness becomes remarkable, leaving the width almost unchanged as a plane-strain type of necking (see Fig. 4(b)). Fig. 4(a) and (b) show that, once the NS specimens attain the maximum loading point then strain localization occurs. However, the strain localization process is much depressed in the LTS specimens during a long straining stage between $\Delta L / L_{0}=0.06$ and 0.21 . It will be shown later that, this macroscopic behaviour has similarities with the trend of void development shown in Fig. 6 for the LTS specimens.

\subsection{Instability characteristics of the two sets of test sheets}

The two types of sheets also exhibit much difference in their plastic instability characteristics. After the load attains its maximum value in the NS specimen, two crossed shear bands appear within the gauge length. The angle between the shear band and the tensile axis is approximately $60^{\circ}$. Afterwards, these shear bands rapidly develop into one or two visible localized necks with further straining, but the deformation outside the localized necks is almost negligible.

Unlike the case in the NS specimens, the instability of the LTS is a much slower process. After the load attains its maximum value, multiple shear bands consecutively appear in the middle region of specimen and form a wide band of instability. The load drops off smoothly with continued straining in tension, while the shear bands spread over the instability region until a diffuse necking of width appears gradually. In this case, the instability band region is almost ten times as wide as that of the localized shear band of NS (see Fig. 5). As the tensile strain keeps on increasing, one of the shear bands becomes more dominant and eventually a marked localized necking occurs.

These characteristics are in accordance with the tensile behaviour recorded in Fig. 4(a) and (b) for the NS 

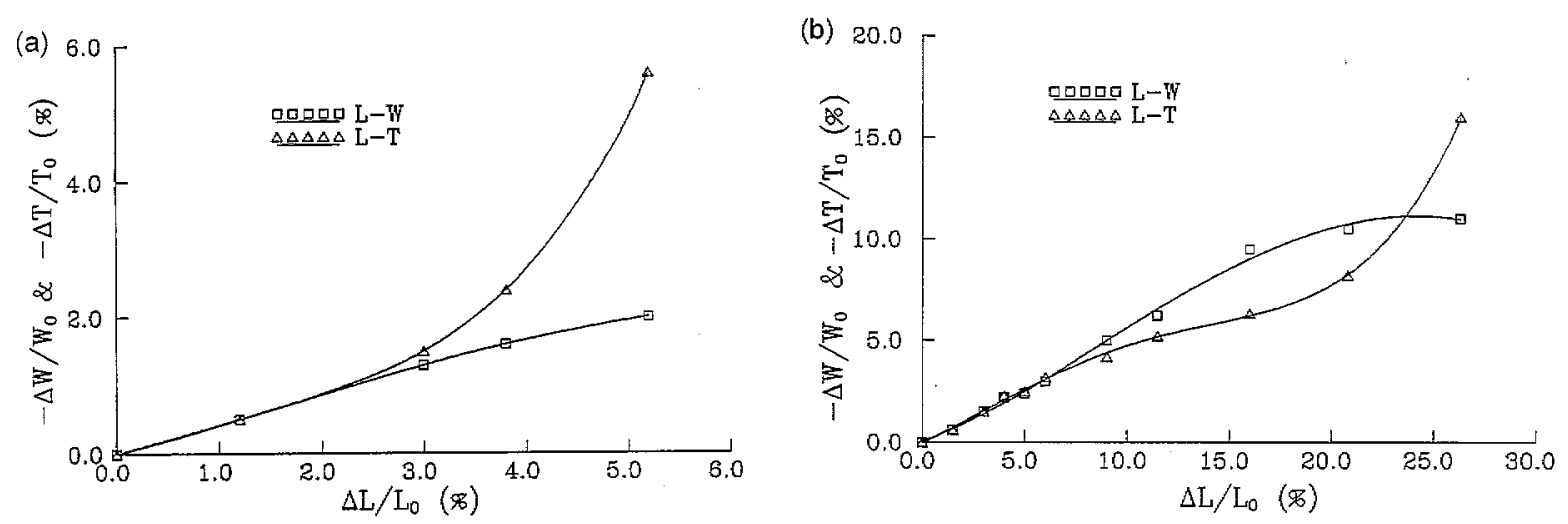

Fig. 4. The variations of sheet thickness and width with respect to engineering strain in the specimens of (a) NS and (b) LTS.

and LTS specimens, respectively. In Fig. 4(a), there is an obvious acceleration of localization in thickness necking, while the sheet width shrinks steadily without observable necking of the diffuse type. The situation changes in Fig. 4(b), there the width variation is larger than that of the thickness during the stage of having $\Delta L / L_{0}=0.06-0.21$. It indicates that the occurrence of diffuse necking takes place first in the LTS and its thickness variation is depressed at this stage. The alternative state comes after $\Delta L / L_{0}=0.21$, then thickness necking is accelerated and localized up to final failure.

\subsection{Evolution and distribution of voids}

Consistent with the previous reports $[8,9]$, the results of the present investigation also indicate that, the fraction of voids has a substantial increase after the occurrence of instability. The variations of void area fraction with respect to the engineering strains were measured for both sets of test sheets. The results are shown in Fig. 6, each data point is the value averaged over the results measured on eight micrographs (magnification $500 \times$ ) taken along the thickness section at each sampling location (see the demonstration in Fig. 2). After attaining the maximum load, an abrupt increase in void area fraction occurs immediately in the NS specimens while only a gradual increase can be seen in the LTS specimens. During the strain stage of $\Delta L / L_{0}=0.06-$

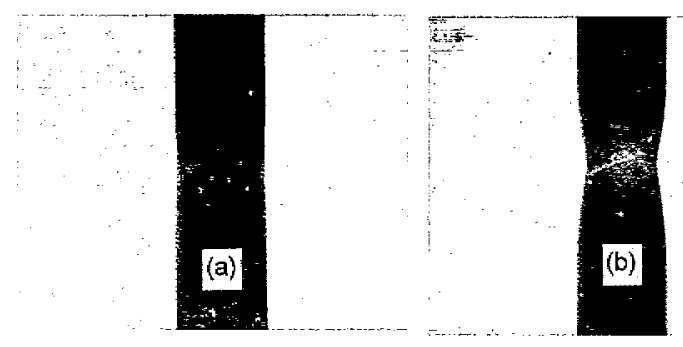

Fig. 5. Local instability characteristics in the specimens of (a) NS and (b) LTS.
0.21 , the increase rate of void area fraction slows down obviously in the LTS, as shown in Fig. 6. This phenomenon is in accordance with the deformation characteristics demonstrated in Fig. 4(b), i.e. the depression of the localizing process in thickness is associated with the temporary inhibition of void development. Comparing the trends of the curves depicted in Fig. 4(b) and 6, we can clearly see the coincidence between the two matters. This is the reason that the leading role of thicknessthinning in Fig. 4(a) give way to the shrinking of width in the LTS specimens of Fig. 4(b) during the straining stage of $\Delta L / L_{0}=0.06-0.21$, until the onset of localized neck, then the LTS has also rapid growth of voids as is the situation in the NS.

Fig. 7 shows the variations of void area fraction with respect to the distance away from the centre of localized neck. In order to have the comparisons on the same basis, both the NS and the LTS specimens are chosen to be at the moment $\left(\Delta L / L_{0}=0.04\right.$ in the NS and $\Delta L / L_{0}=0.21$ in the LTS) when the localized neck is visible. In the region of localized neck, the void area fraction of the two sets of test sheets is approximately

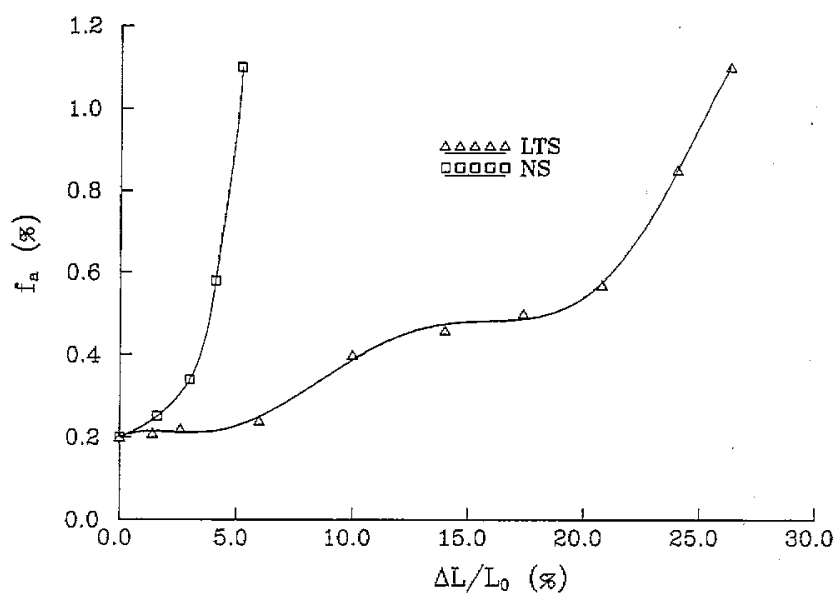

Fig. 6. The variations of void area fraction with respect to engineering strain in the specimens of NS and LTS $\left(f_{\mathrm{a}}\right.$ vs. $\left.\Delta L / L_{0}\right)$. 


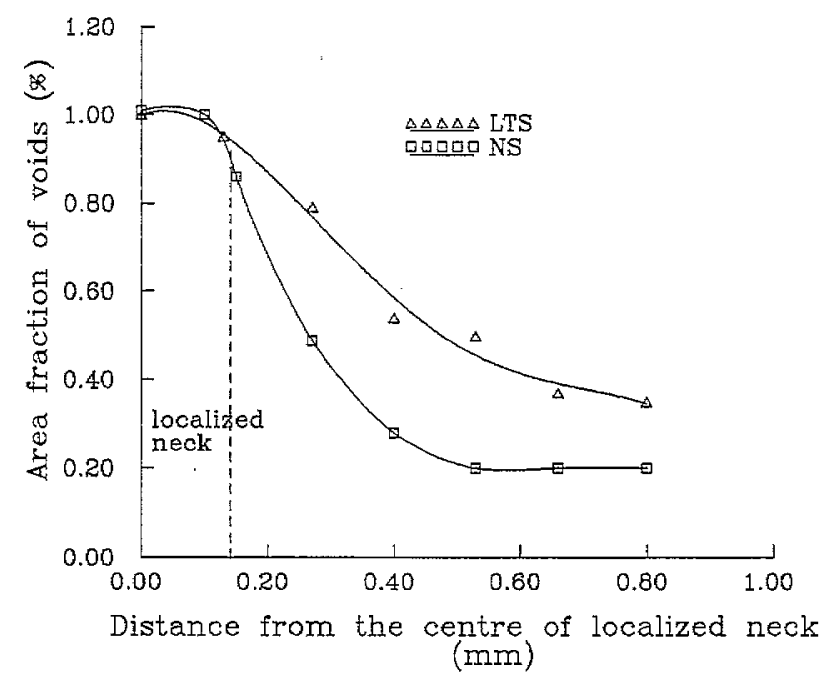

Fig. 7. The distributions of yoid area fraction with respect to the distance $(\mathrm{mm})$ away from the centre of localized neck $\left(\Delta L / L_{0}=0.04\right.$ in NS and $\Delta L / L_{0}=0.21$ in LTS).

$1 \%$, which is possibly the largest amount of void percentage that can be reached in this material. Passing away from the centre of localized neck, the void content of the LTS specimen decreases much slower than that of the NS. The figure indicates that the development of voids is limited within a narrow region of the NS specimen, but, on the contrary, voids nucleate and grow in a much broader region of the LTS and therefore we see wide banding of damaged region in Fig. $5(\mathrm{~b})$.

\subsection{Morphological features of the LTS}

Using Hitachi S-570 SEM equipped with a tensile stage, in situ tensile tests. were performed to observe both the microstructural variation and the morphological condition on the surface of LTS specimen. During the stage of uniform deformation, the number of slip bands increases gradually, but high concentration of these bands are seen piling up at the edge of the microcrater (see Fig. 8 at $\Delta L / L_{0}=0.05$ ). This observation proves that strong strain-hardening effect caused by the microcraters does exist and would therefore resist against the plastic deformation to flow in their surrounding material.

It is also worth noticing that, although the deformation becomes large and concentrated in the middle part of the specimen after attaining the maximum load of the LTS, the appearance of the microcraters are still able to be identified (see Fig. 9 at $\Delta L / L_{0}=0.06$ ). This picture indicates that, the reloading strains have not had the specimen surface recovered from the prestrains imposed by texturing process and hence the microcraters keep on serving as hard blockage obstructing the plastic flow in the material near to the surface of LTS.

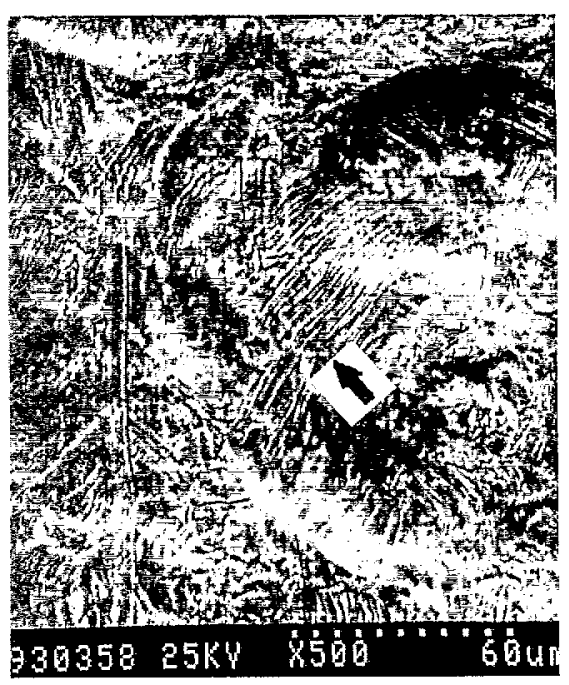

Fig. 8. SEM micrograph on the high concentration of slip bands piling up at the edge of the microcrater on the surface of LTS $\left(\Delta L / L_{0}=0.05\right)$, as shown by arrow.

\section{Discussion}

As mentioned above, the two sets of test sheets, which are cold rolled by using the laser-textured rolls and the normal sanded rolls respectively, exhibit different plastic instability behaviour. The experimental results presented in this paper show that, strain localization is liable to occur in the NS specimens, but the process of this localization is much postponed in the LTS specimens.

According to the geometrical profile of the microcraters (the depth and width of the valley indented on the surface of the LTS are 5 and $40 \mu \mathrm{m}$, respectively). It can be estimated that the prestrain imposed on the

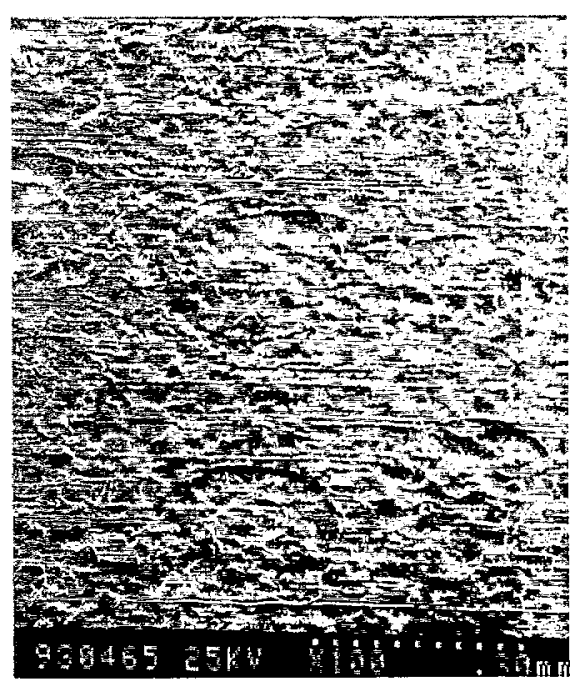

Fig. 9. The surface feature of the LTS with distinguishable microcraters after attaining the maximum load $\left(\Delta L / L_{0}=0.06\right)$. 
surface may come up to an average amount of 0.04 . Another statistic is that the total area of the microcraters occupies $30 \%$ of the sheet surface. A prestrain of 0.04 existing in such a substantial proportion of surface area would act as a strain-hardening spot in stabilizing deformation, as is justified qualitatively in Section 3.4 of this paper. It is well understood that, associated with the proceeding of plastic deformation, geometrical or material inhomogeneity develops gradually and unstable flow would first occur in some weak region. The hardening spots are likely to resist the deformation in the adjacent regions where the instability initiates, then the deformation cannot flow freely but rather shift to other weak regions, and so on. Hence, the instability deformation spreads over an extensive region as seen in Fig. 5(b) and rapid development of localized necking is inhibited in the LTS specimen. It is reasonable to think that these hardening spots have an effect of sharing out deformation among their neighbouring materials.

In addition, the difference in voiding of the two types of sheets tested also reflects their instability characteristics. The behaviour observed in the NS specimen indicates that, on the one hand localization triggers fast development of voids and on the other hand rapid increasing of voiding accelerates the instability process. This is in accordance with the main findings $[9,10]$ obtained by theoretical analysis and microstructural observation for the instability problem in plane sheet. It is found that the rate of voiding is a controlling factor which favours shear-band bifurcation, and on the other hand, bifurcation promotes further voiding and localization. Therefore, in the situation where there are some means to depress either the development of localization or the rate of voiding damage, such as using the hardening agents in the LTS, and we do see the depressing effect as demonstrated by the two void growth curves depicted for the NS and LTS specimens in Fig. 6 , then the material can be much stabilized by delaying bifurcation phenomenon.

Further study is definitely necessary to properly adjust the factors which are controlling the laser-texturing technique, so as to obtain the best stabilizing effects from those microcraters indented on sheet surface. We would expect that, with a certain extent of properly cold rolling reduction, the larger the cold rolling reduction is applied the better the additional hardening effects of microcraters can be obtained. Consequently, more improvement of ductility is gained for steel sheets. In this paper, a $12.5 \%$ reduction of cold rolling is adopted to enhance the prospective effects, while the effect is negligible when a $1 \%$ reduction of cold rolling is applied. The $12.5 \%$ reduction induced a prestrain of 0.04 in the microcraters, as estimated previously, this amount of prestrain leads the material towards the strength limit of the NS sheet in Fig. 3. However, in the case of having a $1 \%$ reduction, it can only induce a prestrain of an order lower, such that the material would be just passing the yielding point. The two extremes clearly demonstrated the important role played by the prestrain for forming strain-hardening spots in stabilizing deformation.

\section{Conclusions}

The mechanical and microstructural effects of lasertexturing on steel sheet have been investigated in this paper and the following findings have been reached.

(1) Compared with the normal rolled sheet, the lasertextured sheet exhibit different behaviour in plastic instability and has obvious tendency to postpone strain localization.

(2) Both shear banding and the development of voiding are spread to a much wider region in the laser-textured sheet.

(3) The existence of substantial prestrains and high concentration of slip bands in the LTS sheet prove that, the microcraters indented on its surface are acting as hardening spots. These hardening spots share out deformation and inhibit the increasing rate of voiding, and hence favour the extension of material ductility.

\section{Acknowledgements}

The joint support of this work by the National Natural Science Foundation of China, the Chinese Academy of Sciences and the Laboratory for Nonlinear Mechanics of Continuous Media is gratefully acknowledged.

\section{References}

[1] A.E. Bayoumi and R. Joshi, Appl. Mech. Rev., 45 (2) (1992) S158.

[2] Chen Guangnan and Hu Shiguang, Chin. J. Mech. Eng., 30 (2) (1994) 82.

[3] A.K. Ghosh, Metall. Trans. A, 5 (1974) 1607.

[4] A.K. Ghosh, Metall. Trans. A, 8 (1977) 1221.

[5] M. Imanaka, T. Fujiwana, T. Furubayashi, T. Obara, K. Yunoyama and K. Furukawa, Proc. of $15 t h$ IDDRG Congress, Dearborn, 1988, p. 109

[6] G. Monfort, J. Defourny and A. Bragard, Proc. of 15th IDDRG Congress, Dearborn, 1988, p. 91.

[7] R.Müller, Proc. of 15th IDDRG Congress, Dearborn, 1988, p. 99.

[8] Chen Guangnan and Hu Shiguang, Chin. J. Plasticity Eng,, 1 (1994) 31.

[9] Zhu Chen, Hong Youshi and Li Guochen, Mater. Sci. Tech., 9 (1993) 1037

[10] Guo-Chen Li and Chen Zhu, Int. J. Plasticity, 11 (1995) 605. 